\title{
Muséologies
}

Les cahiers d'études supérieures

\section{Nathalie Bondil, directrice et conservatrice en chef du Musée des beaux-arts de Montréal}

\section{Alessandra Mariani}

Volume 6, numéro 1, 2012

URI : https://id.erudit.org/iderudit/1011533ar

DOI : https://doi.org/10.7202/1011533ar

Aller au sommaire du numéro

Éditeur(s)

Association Québécoise de Promotion des Recherches Étudiantes en

Muséologie (AQPREM)

ISSN

1718-5181 (imprimé)

1929-7815 (numérique)

Découvrir la revue

Citer ce document

Mariani, A. (2012). Nathalie Bondil, directrice et conservatrice en chef du

Musée des beaux-arts de Montréal. Muséologies, 6(1), 77-99.

https://doi.org/10.7202/1011533ar

Tous droits réservés (C) Association Québécoise de Promotion des Recherches Étudiantes en Muséologie (AQPREM), 2012
Ce document est protégé par la loi sur le droit d'auteur. L'utilisation des services d'Érudit (y compris la reproduction) est assujettie à sa politique d'utilisation que vous pouvez consulter en ligne.

https://apropos.erudit.org/fr/usagers/politique-dutilisation/ 
Dialogue un

\section{Nathalie Bondil, directrice et conservatrice en chef du Musée des beaux-arts de Montréal}

Alessandra Mariani

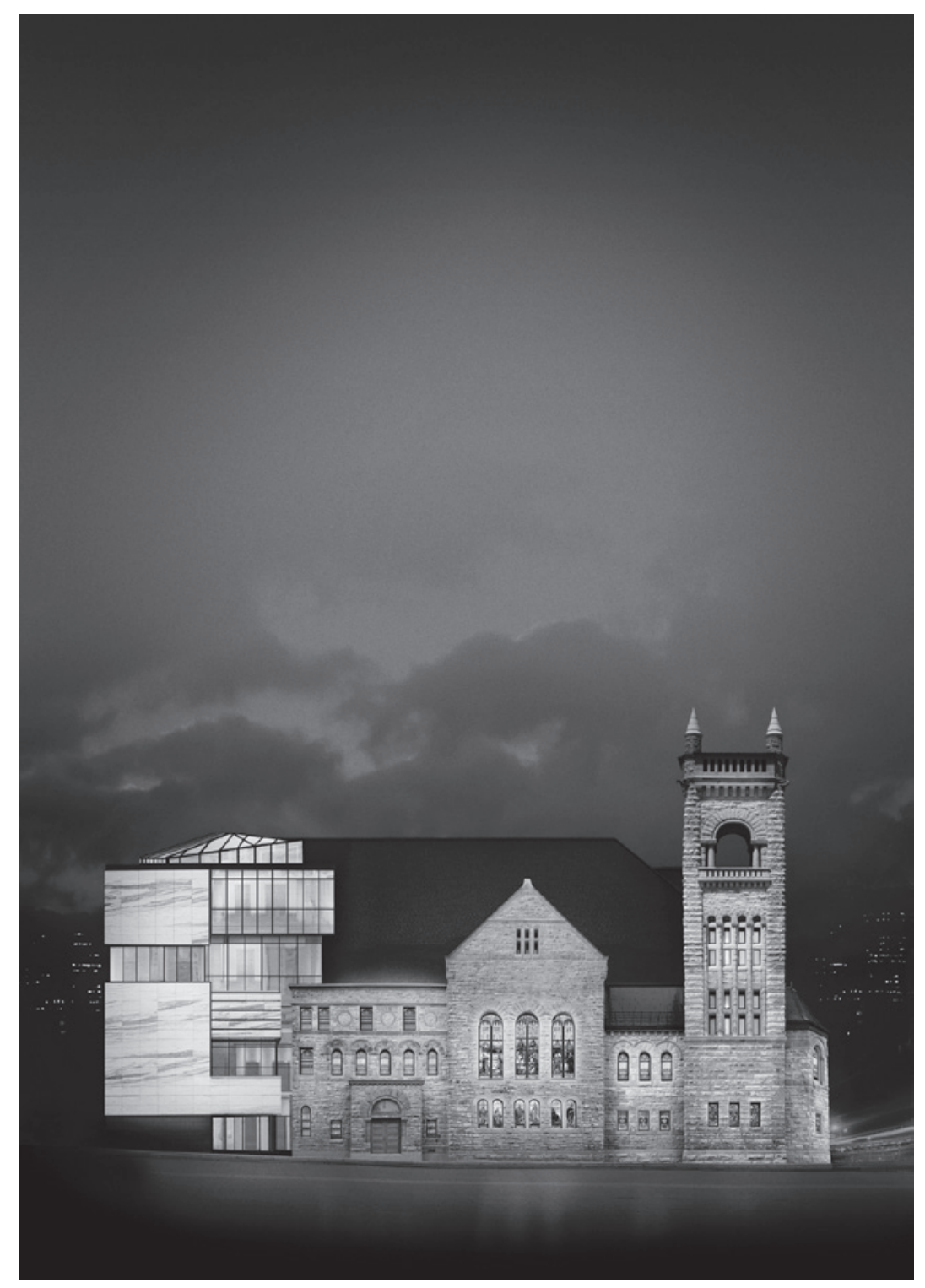

Pavillon Claire et Marc Bourgie Élévation

Photo: Paul Boisvert 
Franco-canadienne, historienne de l'art de formation et diplômée de l'Institut national du patrimoine de Paris, Nathalie Bondil est conservatrice en chef depuis 2000 et aussi directrice, depuis 2007, du Musée des beaux-arts de Montréal (MBAM). Elle a été commissaire notamment des expositions suivantes: Hitchcock et l'art, Picasso érotique, Catherine la Grande, Van Dongen, Maurice Denis et Cuba: art et histoire depuis 1868 à nos jours. Depuis qu'elle dirige le MBAM, elle a invité dans sa programmation décloisonnée la mode (Yves Saint Laurent et Jean Paul Gaultier) et la musique (Warhol Live, Imagine, We Want Miles et Feininger), étendu la politique de gratuité à l'art contemporain (de E-art à Big Bang) et exporté un nombre record de ses "expositions maison" vers l'international. En parallèle, le musée s'est intégralement redéployé et agrandi, avec l'ajout d'un nouveau pavillon d'art dédié à l'art québécois et canadien ainsi que d'une salle de concert. Elle a entrepris un vaste programme de publications scientifiques sur les collections, lancé une grande campagne de dons d'œuvres et initie maintenant un chantier majeur lié à l'éducation scolaire et universitaire.

Au moment où ces lignes allaient sous presse, le Musée a atteint de nouveaux sommets pour les dons d'oeuvres, la fréquentation et le volume d'adhésion. 
Madame Bondil, quel chemin vous a menée au Musée des beaux-arts de Montréal? Comment votre intérêt pour les musées s'est-il développé?

NB La Méditerranéenne que je suis n'aurait jamais imaginé vivre à Montréal il y a treize ans. D’origine provençale, née à Barcelone, ayant vécu au Maroc, j'ai toujours privilégié les voyages dans le temps et dans l'espace, l'histoire de l'art étant une discipline ouverte à ces deux dimensions. L'École du Louvre, à Paris, d'où je suis diplômée, offrait un double avantage: les cours qui y sont dispensés exigent une solide connaissance des collections, basée sur un cursus d'histoire générale de l'art exhaustif. J'ai visité d'innombrables musées, cette expérience pédagogique formant un goût pour l'objet. Je souhaiterais d'ailleurs renforcer au MBAM ce type de programme en collaboration avec les universités, d'autant plus que le musée possède une collection généraliste appropriée. J'ai réussi le concours de l'Institut national du patrimoine; en France, cette grande école d'État est le passage obligé pour quiconque veut travailler comme conservateur dans un musée. Spécialisée en sculpture et en art du XIX ${ }^{\mathrm{e}}$ siècle, c'est au Musée des monuments français - aujourd'hui Cité de l'architecture et du patrimoine au palais de Chaillot - que j'ai occupé un premier poste. Guy Cogeval, qui m’avait embauchée et qui avait été l'un de mes professeurs, m'offrit par la suite de le seconder à Montréal.

Si je dois ma formation formelle à la France, j’ai découvert au Québec, grâce au MBAM, des valeurs, tant professionnelles que citoyennes, qui m'ont profondément touchée. J'ai voulu partir à quelques reprises, mais les projets et les enjeux qui m'étaient à chaque fois proposés m'ont finalement convaincue de rester; et j'ai été nommée à la direction du musée il y a cinq ans.

\section{Quels outils ont façonné votre pratique en tant que directrice générale? \\ NB Une formation universitaire solide en muséologie complétée par} une expérience de terrain intense et variée à titre de conservatrice en chef pendant des années et de commissaire de plusieurs expositions de nature complexe. Par ailleurs, la liberté, le rythme et l'ambition soutenus de la programmation, l'éventail encyclopédique et pluridisciplinaire de l'institution permettent d'accumuler rapidement une expertise. Ensuite, des raisons "politiques" (le citoyen ou politikos) et mes convictions "républicaines" (res publica ou affaires publiques) motivent mon engagement auprès d'un service public: si ce discours est français, en réalité, c'est dans cette société que j'ai trouvé, au travers du prisme du musée, les valeurs qui nourrissent l'appareil collectif, philanthropique et culturel. Je le pense sans idéalisme mais sans négativisme: la culture est ici une cause essentielle, une nécessité de survie, et non une culture ostentatoire, achetée ou vécue par procuration. De plus, elle est aujourd'hui au défi de se définir dans un multiculturalisme stimulant. Au prorata d'une population somme toute modeste en nombre, la force de créativité qui s'en émane en témoigne incontestablement. Havre plus que ghetto, Montréal est une ville américaine et européenne, multiculturelle et multiethnique, une métropole affranchie, décomplexée, en un mot créative. 


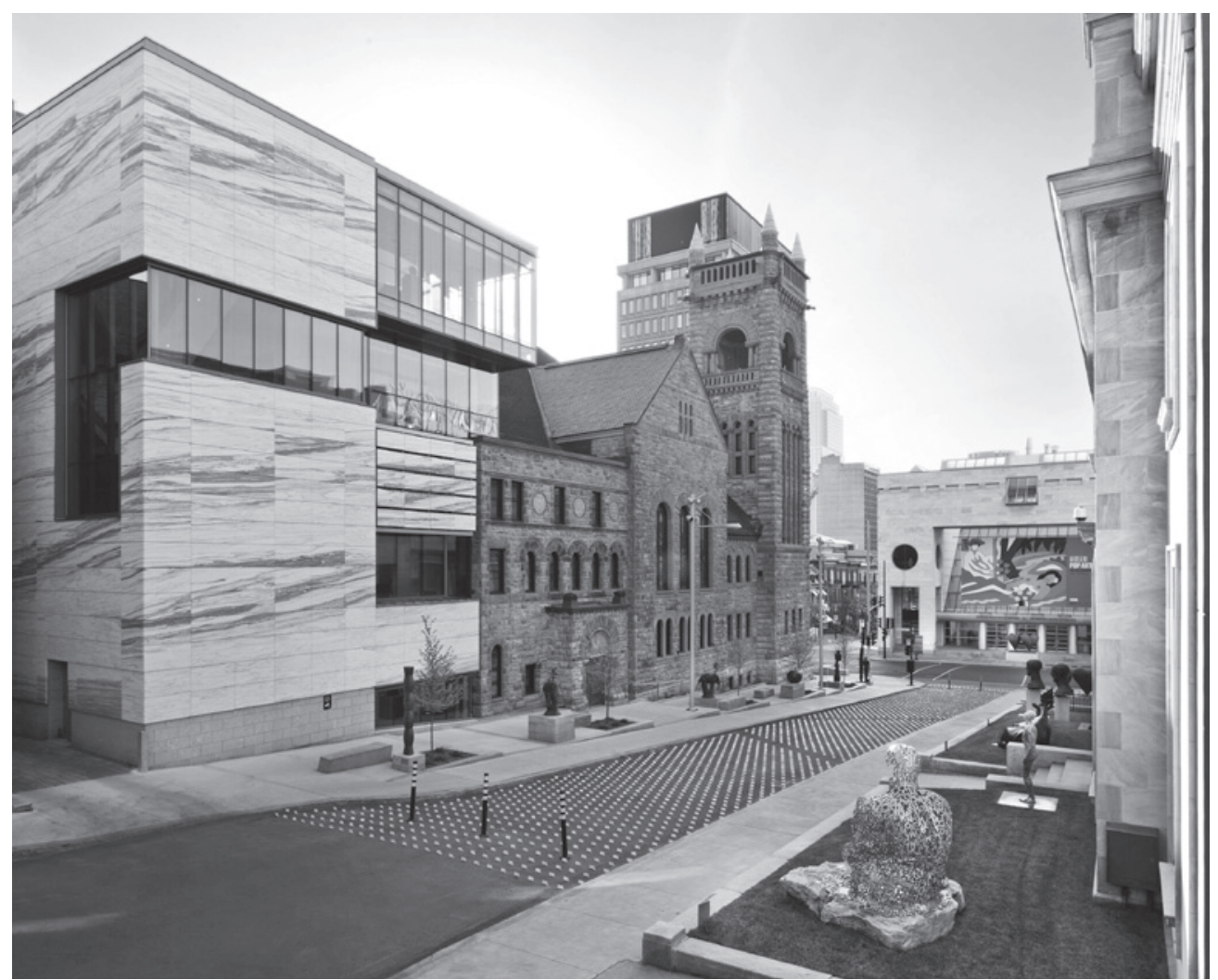

Jardin des sculptures

Installation de Claude Cormier, 2012

Photo: Michel Dubreuil 


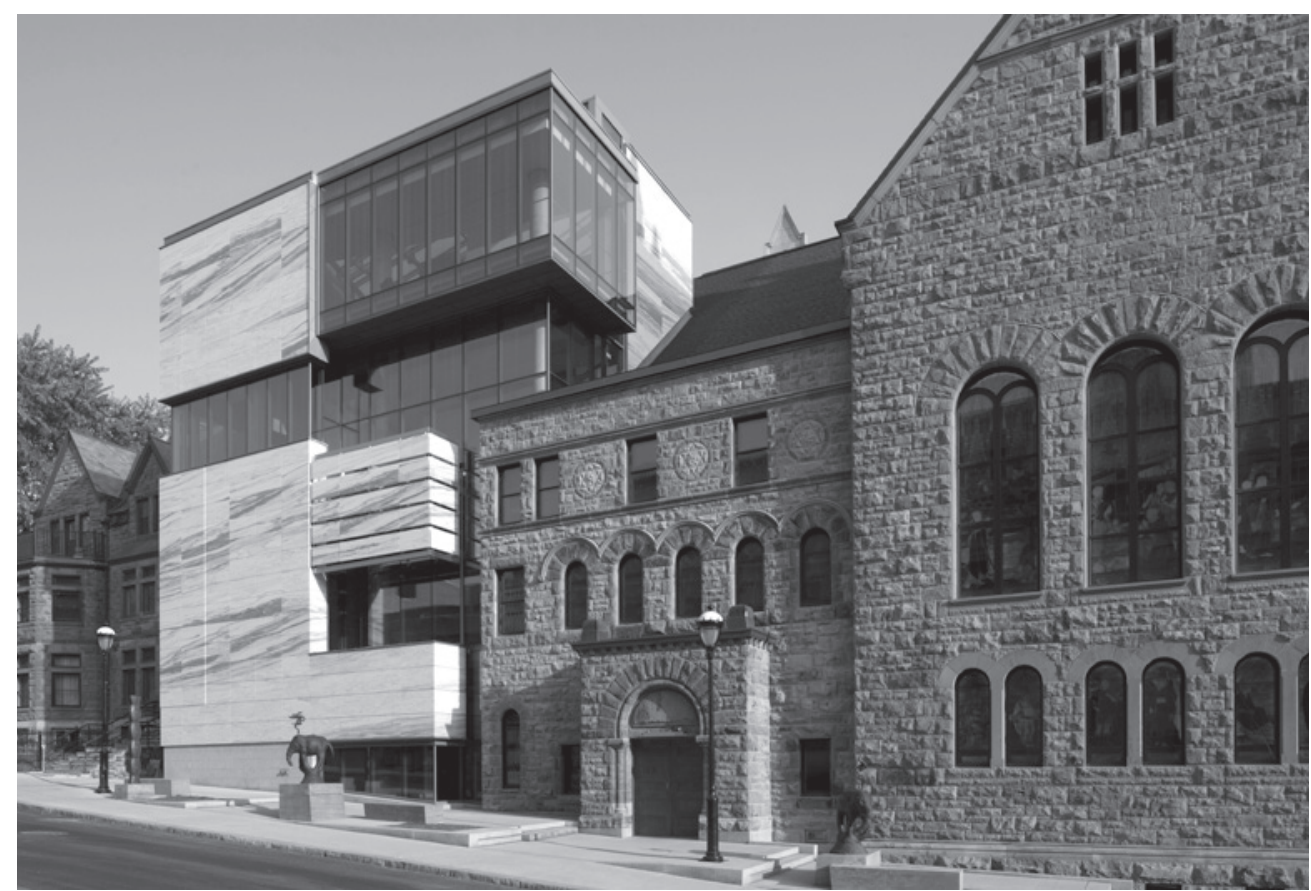

Pavillon Claire et Marc Bourgie

Photo: Denis Farley

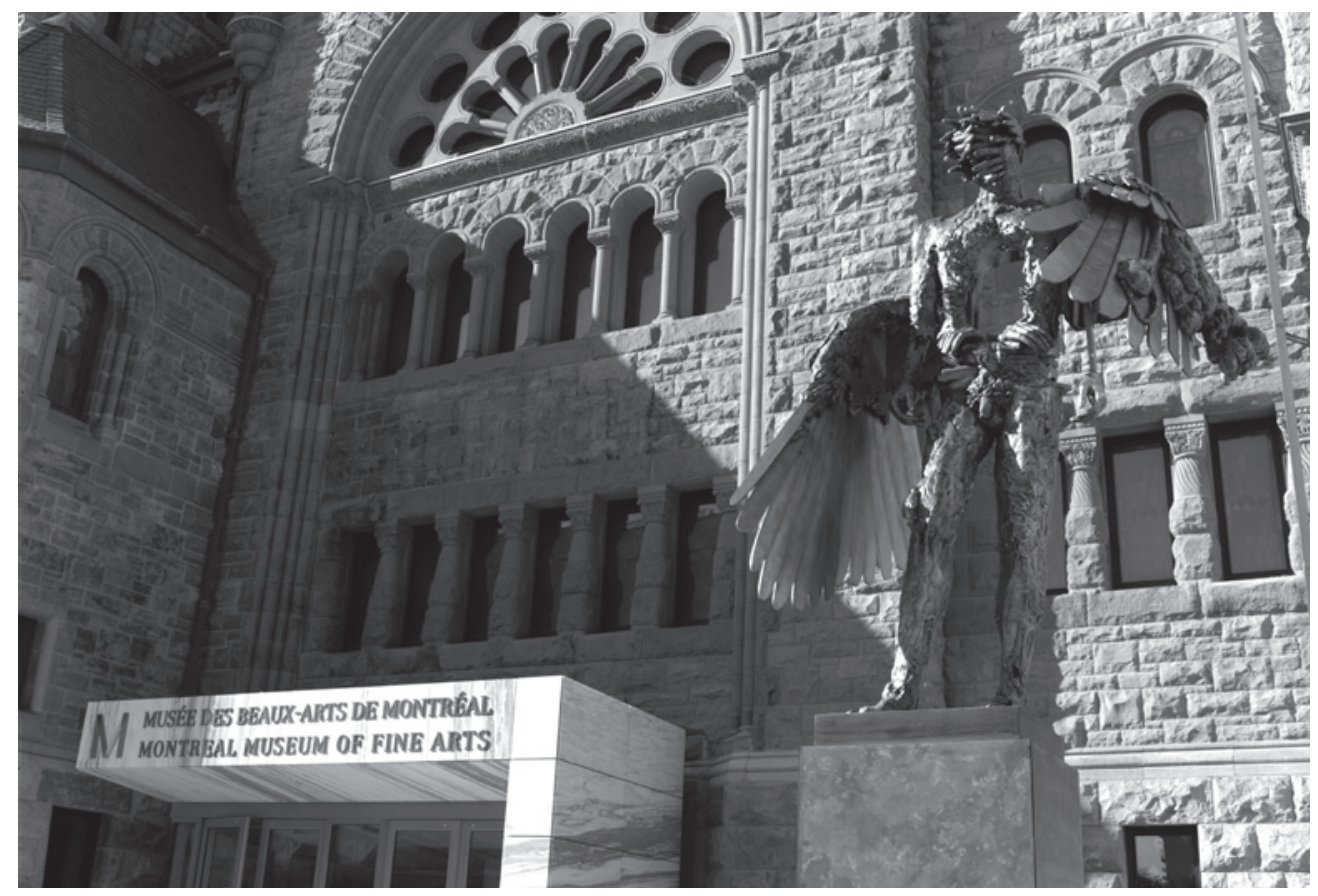

L'œil de David Altmejd

Photo: Bernard Fougères 
Évidemment, ce contexte est gratifiant pour qui veut investir le champ culturel, car les attentes sont réelles et les possibilités d'agir pour changer aussi. «Je est dans nous": cette intuition est devenue une ambition.

Le Musée des beaux-arts de Montréal vit, de toute évidence, des moments forts depuis votre nomination: expositions à grand déploiement réussies, ouverture de l'aile de l'art québécois et canadien, création de la salle de concert Bourgie, redéploiement des collections, accroissement des expositions d'art contemporain, diversification des acquisitions, etc. Pouvez-vous nous en dire davantage sur ce renouveau du musée?

NB Mes cinq années à la direction du MBAM se lisent dans la continuité d'efforts individuels et collectifs depuis 150 ans -le musée étant créé en 1860 -, une durée assez remarquable à l'échelle continentale. La nouvelle aile contribue à renforcer une identité plurielle et singulière, qui a fait de cette institution le plus grand musée d'art du Québec. Cet ensemble architectural hétéroclite, construit pavillon par pavillon, avec ses collections devenues encyclopédiques, résulte d'une croissance progressive. Mise en abyme de la ville, cet étonnant complexe patrimonial, avec sa collection d'édifices, offre un résumé de son histoire architecturale. Cette dimension est aujourd'hui renforcée par un jardin de sculptures agrandi, qui délimite ses frontières, les cœurs de Jim Dine inscrivant le centre emblématique d'une cité muséale qui peine encore à trouver une image synthétique... quand son nom plus approprié serait "Les Musées des beaux-arts»! Porté par une action volontariste, le musée a grandi en toute liberté, sans mandat restrictif, se développant au gré des passions et des donations qui l'ont édifié. Selon la terminologie anglo-saxonne, cette gallery a d'abord collectionné les beaux-arts européens, puis canadiens. À ceux qui s'étonnent encore de notre implication en art actuel, je rappelle que, depuis les Salons du printemps et la création d'une école d'art, les artistes ont toujours été très présents au musée: rien de plus normal puisque la mission muséale se légitime dans la durée. Nos contemporains d'hier sont nos modernes d'aujourd'hui, et je tiens à garder ce pouls bien vivant pour demain. À partir de 1916, un virage décisif vers une collection de type museum a ouvert le champ vers les cultures du monde et les arts décoratifs, permettant de rapprocher puis d'intégrer la remarquable collection du Musée des arts décoratifs. Toutes proportions gardées, cette évolution de notre "Pocket MET» [Metropolitan Museum of Art de poche] peut être comprise en parallèle historique avec la nature encyclopédique développée par le Museum of Fine Arts de Boston ou le MET de New York.

L'extension vers l'église avait été envisagée, avant même l'ouverture du pavillon Desmarais en 1991, par notre précédent président, Bernard Lamarre. Un temps imaginé comme un pavillon bibliothèque puis comme un pavillon du patrimoine religieux, il nous est apparu qu'il fallait y exposer l'art québécois et canadien indignement sous-représenté. L'effet domino m'a permis de redéployer les arts décoratifs et le design, de repenser le parcours des maîtres anciens jusqu'à l'art moderne, de développer le territoire des cultures du monde, enfin de dynamiser l'art contemporain dont les formats imposants nécessitent des espaces généreux et de fréquentes rotations. J'ai toujours défendu une vision 
globale du musée : il fallait embrasser la variété des collections qui fait sa richesse et son unicité à l'échelle canadienne; chacune gagnait ainsi en lisibilité, et donc en monumentalité, parce que mieux circonscrite dans un bâtiment. Grâce à cette expansion, chaque département trouvait son centre de gravité par l'entremise d'un espace pavillonnaire. C'est la raison pour laquelle je voulais éditer non pas une seule publication scientifique sur l'art québécois et canadien, mais trois volumes sur toutes les collections pour établir de façon éloquente leur importance et leur singularité.

Bien entendu, le nouveau pavillon d'art québécois et canadien Claire et Marc Bourgie s'affirme comme le fer de lance, attendu et nécessaire, de ce redéploiement intégral, mais ces 600 œuvres s'inscrivent dans un vaste corpus de 4000 objets aujourd'hui restaurés, étudiés et réinstallés: il ne s'agit pas seulement de belles couleurs sur les murs! Il s'agit bien d'un chantier à long terme, avec un investissement muséologique s'étalant sur plusieurs années, une première dans l'histoire institutionnelle. Si la sauvegarde de l'église Erskine \& American était au cœur du projet d'agrandissement, offrant une solution plus éthique qu'économique de recyclage architectural, cette réinvention muséale englobait l'ensemble des pavillons. La diversité des collections est un atout qui autorise une variété d'approches muséographiques, donc autant de "sésames" pour faire découvrir ou redécouvrir les œuvres. Les parcours structurés logiquement ont été considérablement enrichis en œuvres et en textes, reflétant le travail de fond qui a été effectué tant au niveau de la recherche que de la restauration et de la scénographie. Dans le pavillon de l'art québécois et canadien, c'est l'histoire d'un pays qui est abordée. La profondeur historique du Québec et l'amplitude géographique du Canada représentées par les œuvres contribuent à la construction d'une monumentalité symbolique, les fils chronologique et thématique de ces espaces se déroulant à la façon d'un «théâtre de la mémoire " pour citer Roland Recht.

La conversion de la nef de l'église est, en fait, le fruit d'une rencontre opportune avec un mécène éclairé, Pierre Bourgie, qui cherchait de son côté à créer une salle de concert pour de petits ensembles. Or cette nef présentait une acoustique excellente et une beauté indéniable avec ses vitraux de Tiffany. La vocation pluridisciplinaire inscrite dans l'ADN du musée comme mes initiatives d'expositions musicales ont permis d'arrimer rapidement nos projets. À l'aide d'un répertoire reflétant l'universalisme des collections et de la programmation, nous avons imaginé avec sa nouvelle Fondation Arte Musica, en résidence au MBAM depuis 2008, un dialogue original et décloisonné pour explorer les liens entre musique et arts visuels : c'est une combinaison originale dans le monde muséal.

La collection de maitres anciens, impressionnistes et modernes, est pour le musée une richesse inestimable, un ensemble unique au Québec et irremplaçable au Canada. Cette collection permet d'aborder une histoire du goût, du sentiment classique, baroque, romantique, la musique complétant l'expérience de délectation. Les arts décoratifs et le design 
constituent pour nous une carte maîtresse pour développer de nouveaux publics parce que leur objet est immédiatement identifiable et qu'il permet des rapprochements transhistoriques, formalistes, techniques et stylistiques. Indispensables fenêtres sur une société multiculturelle, les cultures du monde sont revitalisées présentement grâce à la collaboration de consultants en art précolombien, islamique, asiatique... Finalement, l'art contemporain, international, hybride et métissé est sujet à une présentation renouvelée à chaque année en suivant des thématiques transversales jugées pertinentes, actuelles et nécessaires.

Redonner une lisibilité et une visibilité à toutes les collections... tout en concevant, présentant et souvent exportant une programmation d'expositions d'envergure, avec les mêmes équipes, s'avérait un défi périlleux. L'institution ne pouvant fermer ses portes pour des raisons budgétaires, le niveau de productivité se révélait extrême et la logistique délicate. Heureusement, le sentiment d'appartenance est très développé: le personnel est au service du musée et non le musée au service d'une personne! Des améliorations restent à apporter, d'autres scenarii seront inventés, mais un pas considérable a été fait.

\section{L'expansion des collections est devenue, dans de nombreuses institutions, une zone "délicate». Vous êtes conservatrice en chef du musée: de quelle façon envisagez-vous le développement de vos collections?}

NB Est-ce notre destin inéluctable que d'enrichir les collections pour s'agrandir, sans fin et sans limites? La collection - raison d'être d'un musée - le différencie d'une kunsthalle ou d'une exhibition box. Le développement de l'institution témoigne d'une croissance organique, l'absence de mandat défini ayant forgé au fil du temps, je l'ai dit, un profil encyclopédique. À la tentation de privilégier un domaine plutôt qu'un autre, j'ai toujours cherché à renforcer cette diversité disciplinaire qui cartographie la spécificité, riche et féconde, du musée aux niveaux métropolitain, québécois et canadien. Dans une culture au défi de la mondialisation, c'est à mon avis une priorité et une chance.

L'enjeu est plutôt de répondre à la variété tout en demeurant sélectif et qualitatif. Nos budgets d'acquisition sont entièrement privés (environ 800000 \$ comparé aux 8000000 \$ obtenus du fédéral par le Musée des beaux-arts d'Ottawa), ce qui est restreint considérant le champ panoramique qui nous est imparti. Cet encyclopédisme nécessite de diversifier les expertises: le musée sort gagnant de cet investissement car, sans spécialiste, une collection meurt et les collectionneurs se détournent. C'est pourquoi j'ai fait appel à davantage de consultants. Pour les cultures du monde, les résultats sont une revitalisation des collections avec des dons d'ensemble majeurs. Des partenariats novateurs pallient la modestie de certains corpus : j'ai ainsi expérimenté pour l'art africain un partenariat inédit entre trois fonds d'origine différente (privée, universitaire et muséale). La consolidation de fonds extérieurs et une stratégie de dépôts à long terme sont des solutions face aux prix toujours plus hauts 


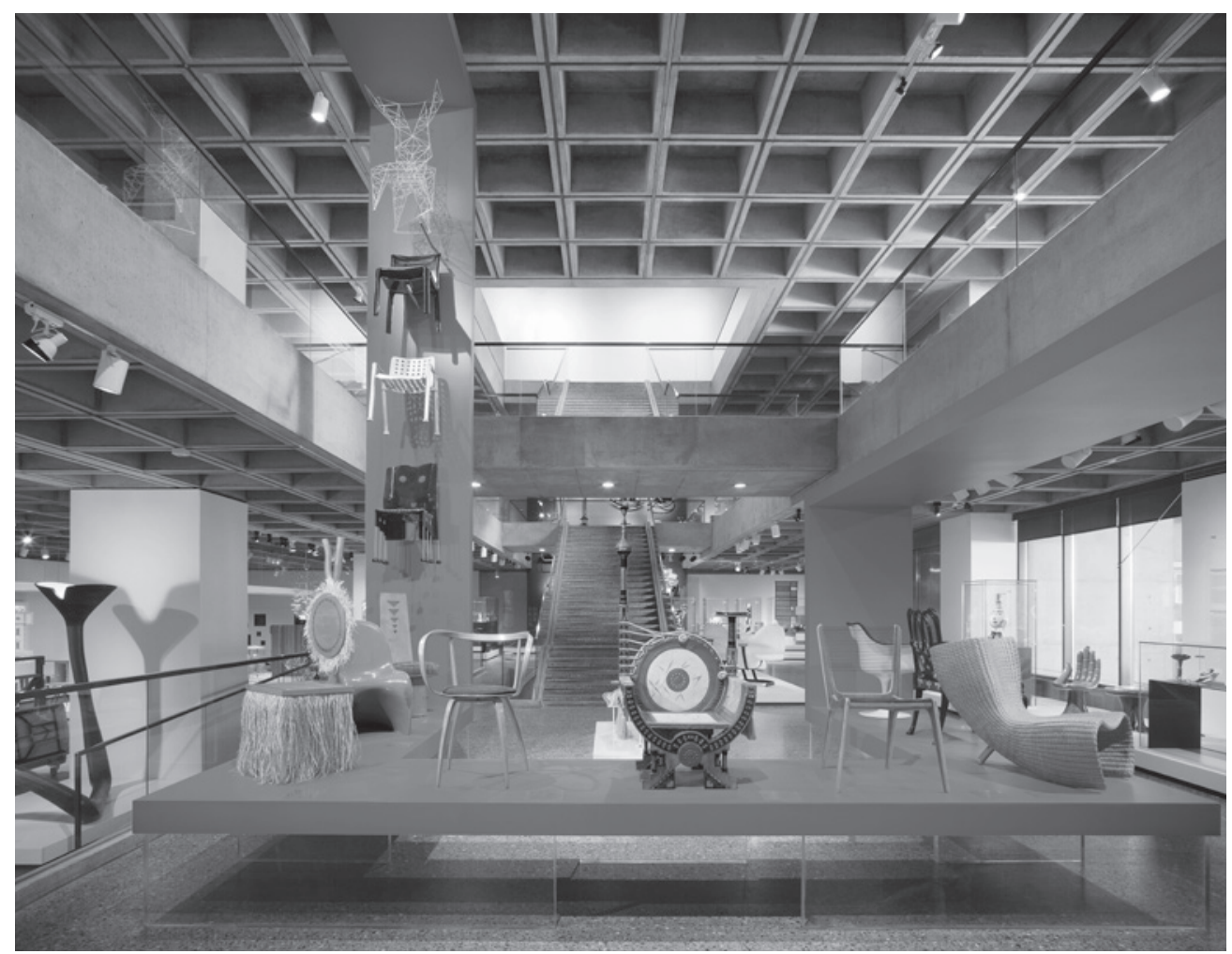

Salle des Arts Décoratifs, 2011

Scénographie : Nathalie Crinière

Photo: Marc Cramer

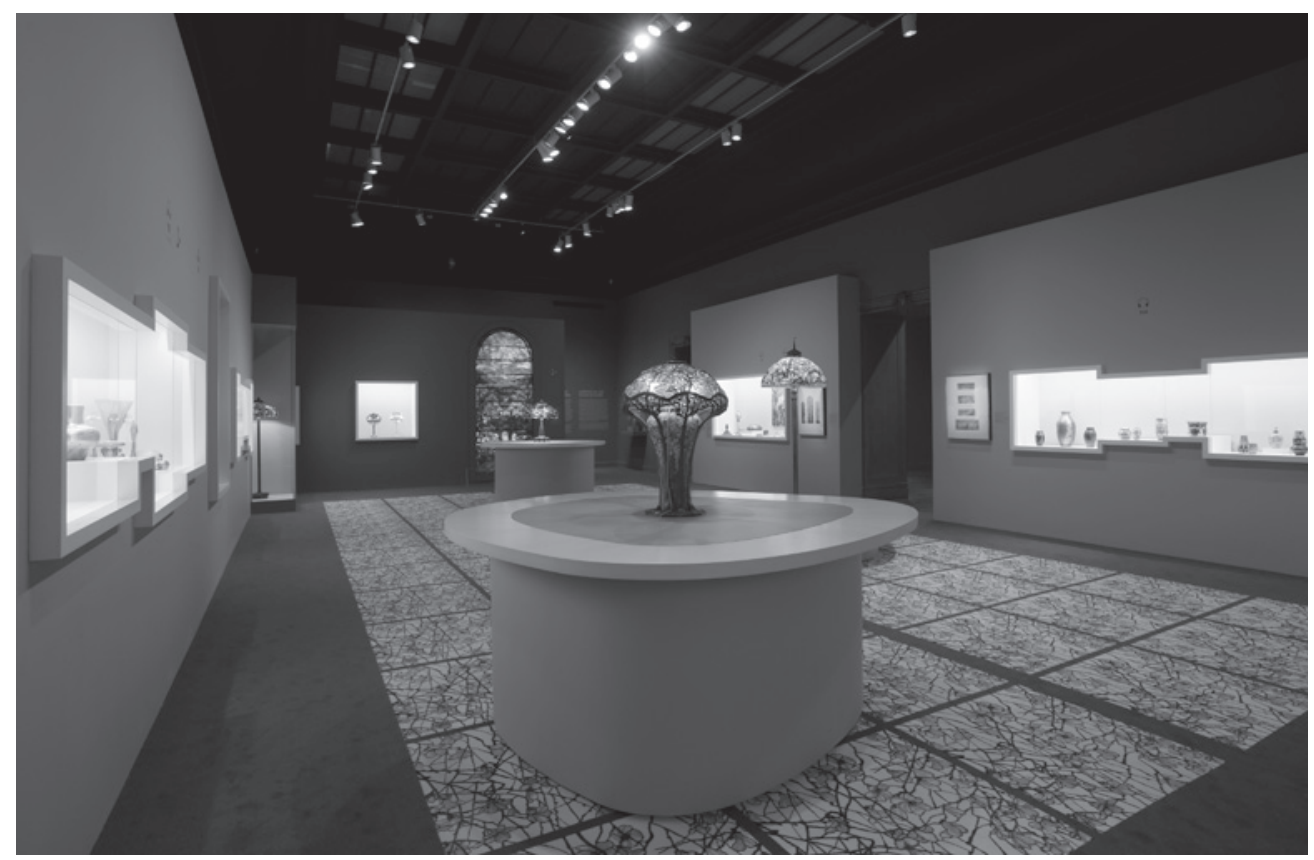

Exposition Le verre selon Tiffany, 2010

Scénographie : Hubert Le Gall

Photo: MBAM, Christine Guest 
d'un marché internationalisé. Une politique d'acquisition en collaboration avec d'autres institutions, des échanges de prêts d'œuvres pour la collection permanente s'avèrent être les stratégies de l'avenir.

Le musée veut refléter la «biodiversité » des collectionneurs. Ces dernières années enregistrent des plafonds inégalés de dons spectaculaires qui s'expliquent par un rapprochement tactique avec cette communauté élargie d'amateurs (que l'on a pu voir lors de l'exposition montée en quelques mois après ma nomination, Pour l'art! Chefs-d'œuvre de nos grands collectionneurs) et plusieurs autres actions, dont le récent redéploiement de toutes les collections. La campagne de dons d'œuvres majeurs pour notre $150^{\mathrm{e}}$ anniversaire se terminera bientôt, avec d'excellents résultats. L'art québécois et canadien est revenu au cœur de notre mission:

l'obtention de grands fonds est toujours possible (l'intégration du musée Marc-Aurèle Fortin), mais malgré des acquisitions remarquables, des lacunes demeurent que nous devons combler. J'ai, par exemple, mis sur pied une collecte de photographies documentaires faites au Québec depuis les années 1960 grâce à l'action éclairée de Marcel Blouin, engagé à titre de consultant; nous avons enfin trouvé un expert pour l'art inuit.... ce qui souligne encore le manque de spécialistes et légitime le besoin de former une relève. Pour l'art contemporain, nous travaillons dans un esprit de complémentarité, et non de compétition, pour éviter les dédoublements avec les autres collections publiques. J'ai aussi initié un parrainage d'artistes avec la Fondation Darling afin de soutenir en amont la création contemporaine. Quant aux arts décoratifs et au design, nous demeurons très actifs: ces objets étant relativement accessibles.

Le plus difficile demeure l'enrichissement de notre collection de maîtres d'art ancien à moderne. Dans un marché mondialisé hautement concurrentiel, chaque œuvre obtenue est une victoire: acquérir récemment un Véronèse, un Lorrain, un Chassériau, un Arp, un Wesselmann est tout simplement extraordinaire. Je rêve d'un bon tableau cubiste depuis des années... Nos œuvres du XIX ${ }^{\mathrm{e}}$ siècle et modernes, une collection modeste à l'échelle des grands musées internationaux, sont sans cesse réclamées par les plus grandes institutions: seulement trois salles du musée concentrent l'essentiel de ces demandes suivant le rythme affolant d'un calendrier d'expositions qui s'intensifie partout dans le monde. Comme le musée est davantage demandeur que prêteur - car il conçoit une majorité de ses projets -, il faut bien réfléchir aux raisons de refuser; très souvent, les œuvres qui nous sont empruntées sont difficilement remplaçables. C'est pourquoi cette collection représente un capital que nous renforçons avec acharnement: acquisition, recherche, publication, promotion... Nous créons des icônes, illustrées en couverture de nos livres, comme pour notre Otto Dix et notre Feininger... La donation Ben Weider d'œuvres napoléoniennes a représenté une occasion formidable qui m'a ouvert les portes vers le champ historique... avec un ambassadeur bien connu dans le monde: le public veut voir le chapeau de l'Empereur! Michal et Renata Hornstein, généreux philanthropes montréalais, sont une bénédiction, car les collectionneurs de ce calibre sont rarissimes au Canada. 
Avec l'équipe, je suis extrêmement déterminée pour toutes les acquisitions: suggérer, soutenir, patienter, convaincre. Il s'agit d'une quête, d'une chasse sans relâche: les prix ne cessent de grimper, les négociations sont ardues et il faut savoir acheter dans des domaines stratégiques. Sur ce point, la générosité des donateurs est cruciale. Le bilan réel de cet actif se fera à long terme: c'est un enjeu de société qui nous transcende, et il nous faut rester modestes car nous sommes, en fin de compte, les bâtisseurs mais aussi les récipiendaires d'un patrimoine.

\section{La programmation s'est transformée à la suite de ce renouveau.}

\section{Pouvez-vous élaborer à ce sujet?}

NB Le musée est reconnu pour sa programmation de grandes expositions, dynamiques, événementielles et novatrices, avec une spécificité pluridisciplinaire qui fait sa notoriété. J'ai élargi cette perspective en renforçant les cultures du monde, en introduisant la mode et la musique. Selon moi, musique et arts visuels font bon ménage, car l'écoute arrête le temps et concentre l'attention: c'est une solution à la consommation frénétique des images qui nous assaillent. L'introduction de la musique spatialisée dans les expositions s'est faite en plusieurs étapes autour d'une trilogie innovante: Warhol Live et Imagine créées par le musée, et Miles Davis du musée de la musique à Paris. Ce projet était assez radical pour un musée des beaux-arts puisque le son du génial trompettiste constituait la colonne vertébrale en autant de salons musicaux pour « exposer " ses chefs-d'œuvre sonores: l'exposition se structurait ainsi autour de l'invisible.

Le son manipule le regard, exerçant un pouvoir tangible sur notre perception. Il requiert des compétences sophistiquées. Nous avons travaillé avec des professionnels chevronnés pour apprendre à maîtriser cette nouvelle dimension. Pour éviter les excès d'effets et les accords artificiels, il y a une précision diabolique à travailler avec des musicologues et autres ingénieurs sonores, un raffinement maniaque qui passe souvent inaperçu du tout-venant. Cette trilogie d'expositions exposait le son comme œuvre participante au contenu et non comme accompagnement, ce que j'ai aussi expérimenté, par ailleurs, avec des audioguides musicaux pour Van Dongen, Tiffany, Waterhouse, avant de les adapter maintenant pour la collection permanente. Il s'agit d'une véritable expertise, extrêmement originale, que je continue de développer avec, entre autres, la Fondation Arte Musica et la nouvelle salle de concert Bourgie. Je suis convaincue que, loin de nous éparpiller, cette expérience synesthésique aide à mieux capter l'attention du visiteur au profit des œuvres : c'est le cas du salon de musique dans l'exposition Lyonel Feininger.

\section{AM Pouvez-vous nous parler de la première exposition que vous avez organisée?}

NB En tant que commissaire et chargée de la coordination générale, Hitchcock et l'art: coincidences fatales en 2001, une exposition novatrice initiée par Guy Cogeval et Dominique Païni, s'est avérée ma première expérience aboutie. Le thème autorisait une grande liberté, chacun étant libre de proposer des rencontres esthétiques pour construire un dialogue décloisonné et poétique. C'était aussi une exposition complexe 


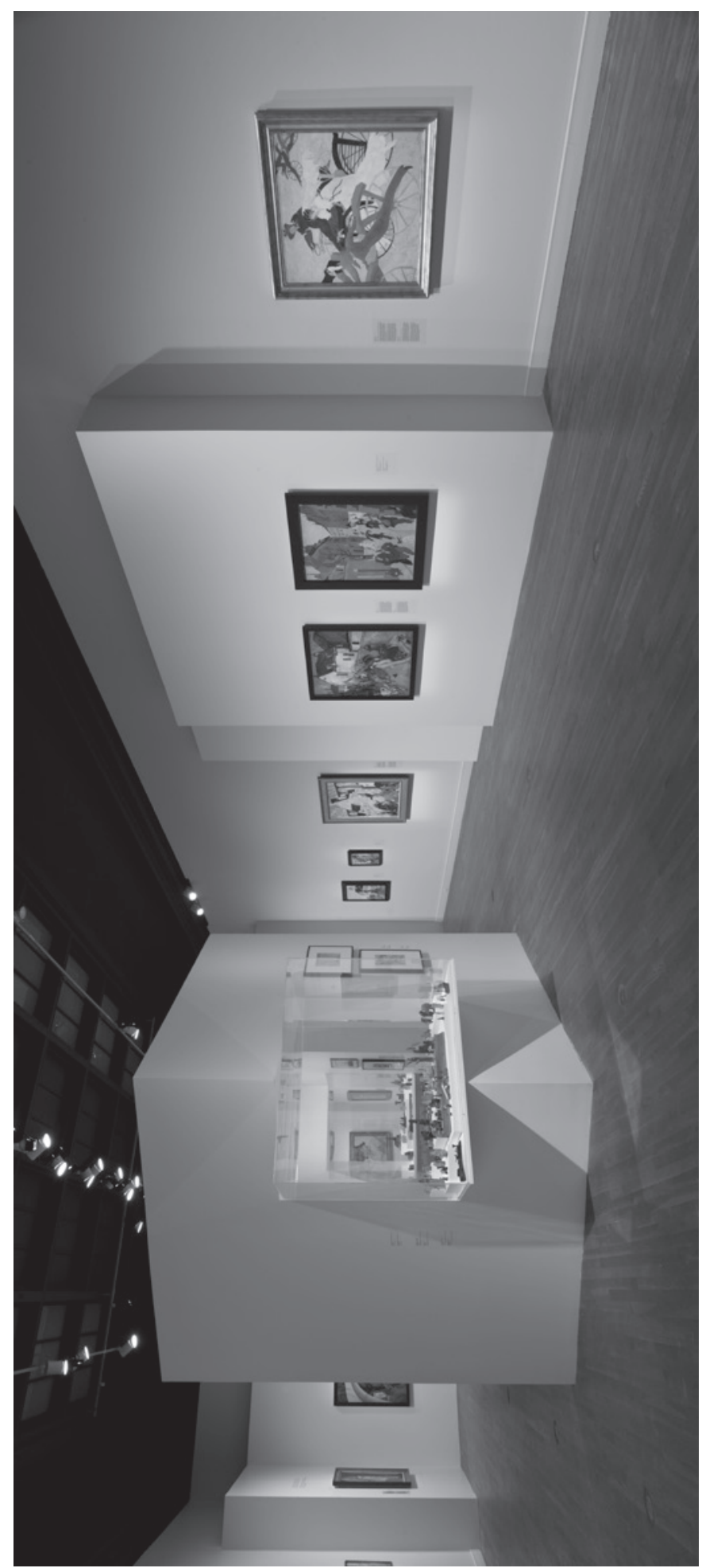

Exposition Lyonel Feininger:

de Manhattan au Bauhaus, 2012

Scénographie: Nathalie Crinière

Photo: MBAM, Denis Farley 


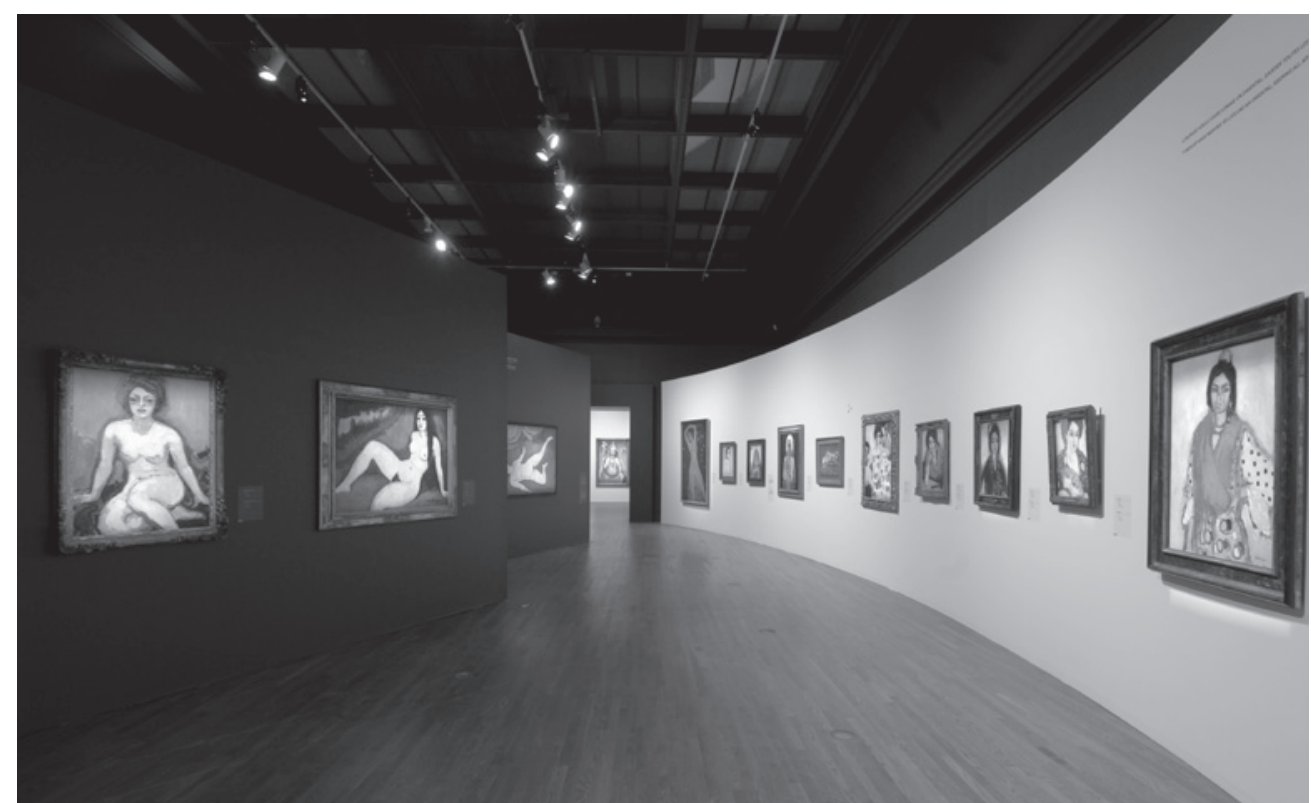

Exposition Van Dongen à Montréal:

un fauve en ville, 2009

Scénographie: Jasmin Oezcebi

(1) Succession Kees Van Dongen/SODRAC (2012)

Photo:MBAM, Christine Guest

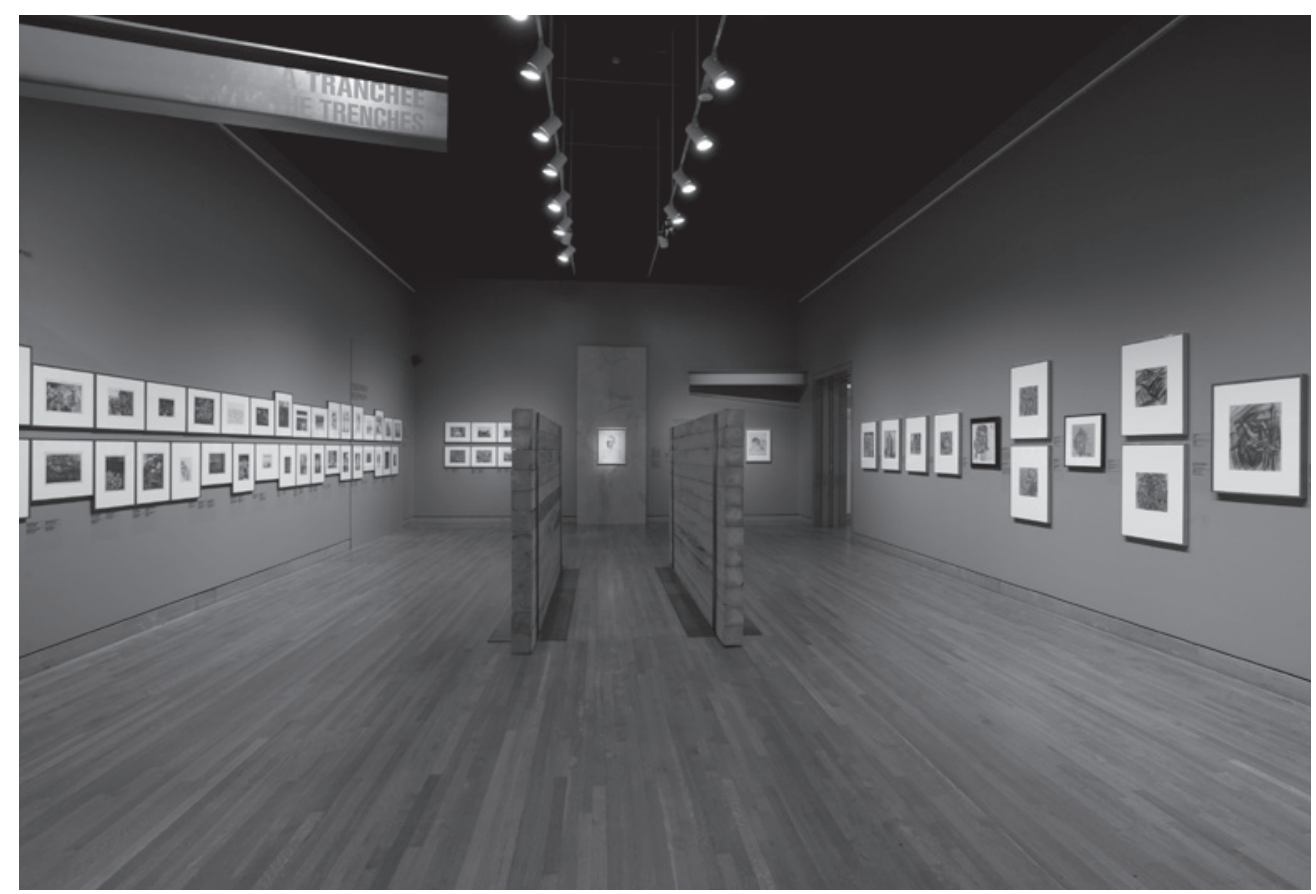

Exposition Rouge Cabaret.

Le monde effroyable et beau d'Otto Dix, 2011

Scénographie : Bruno Braën et Stéphane Roy

(c) Succession Otto Dix / SODRAC (2012)

Photo MBAM, Christine Guest 


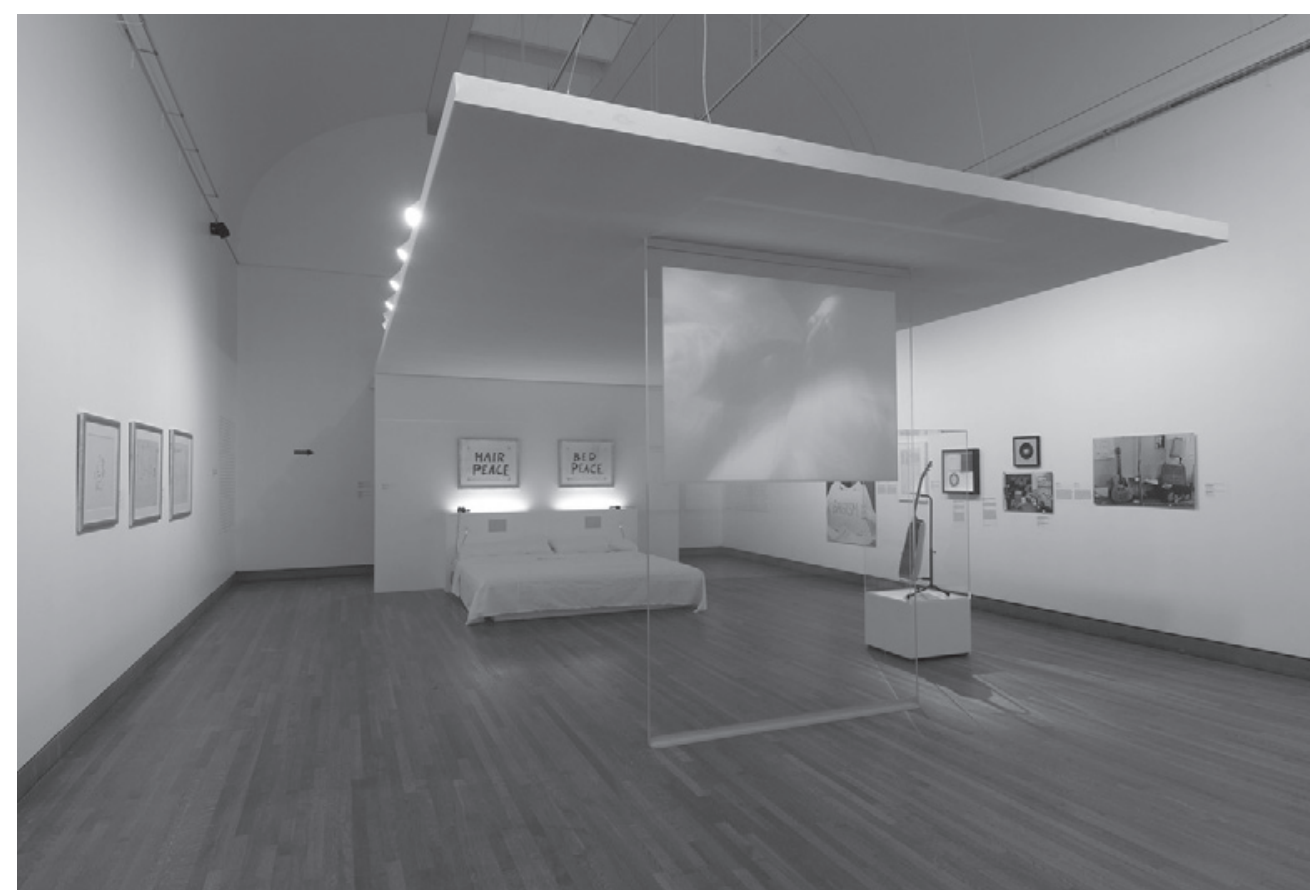

Exposition Imagine.

La ballade pour la paix de John et Yoko, 2009

Scénographie : Boss Design

Photo: MBAM, Brian Merrett

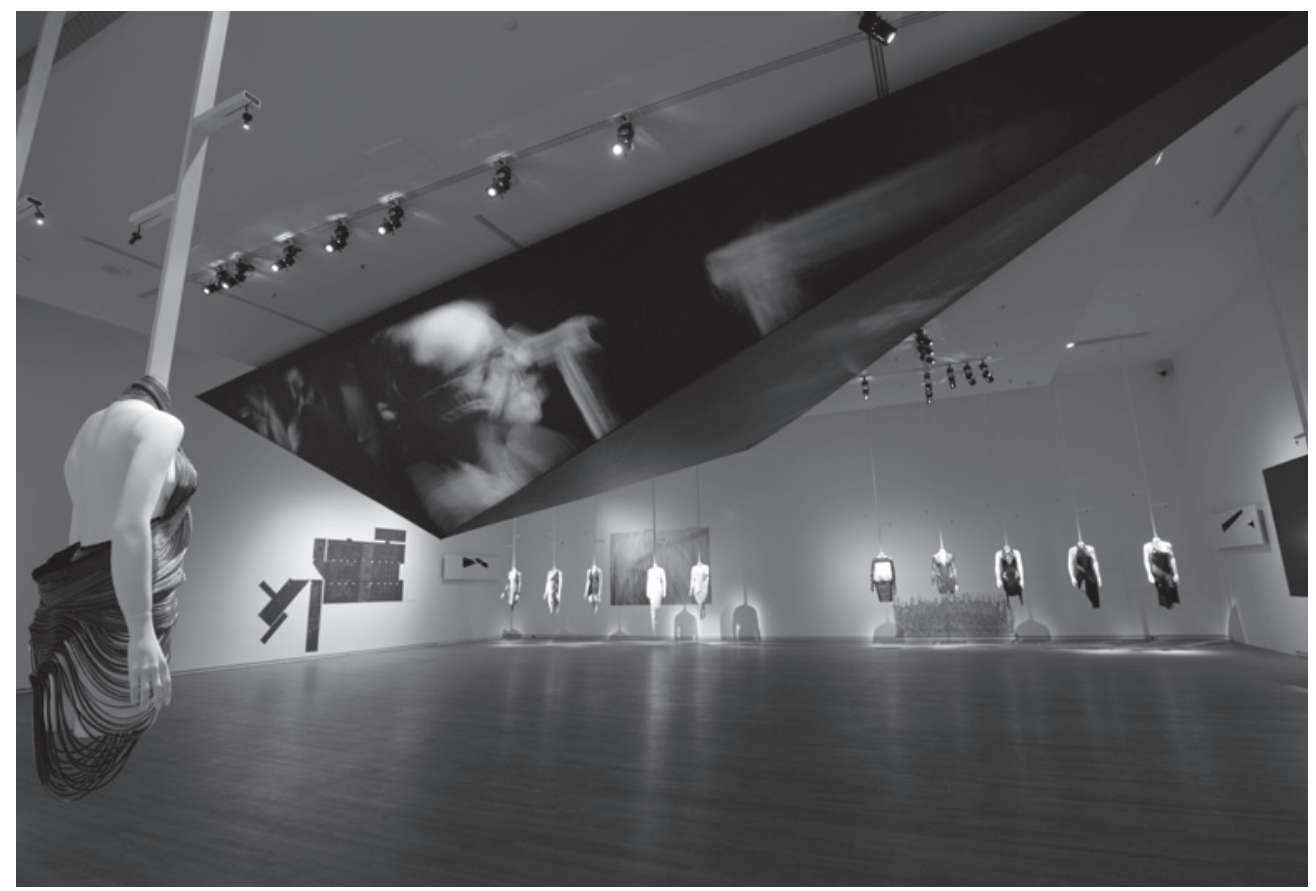

Exposition Denis Gagnon s'expose, 2010

Scénographie: Gilles Saucier

Photo: MBAM, Christine Guest 

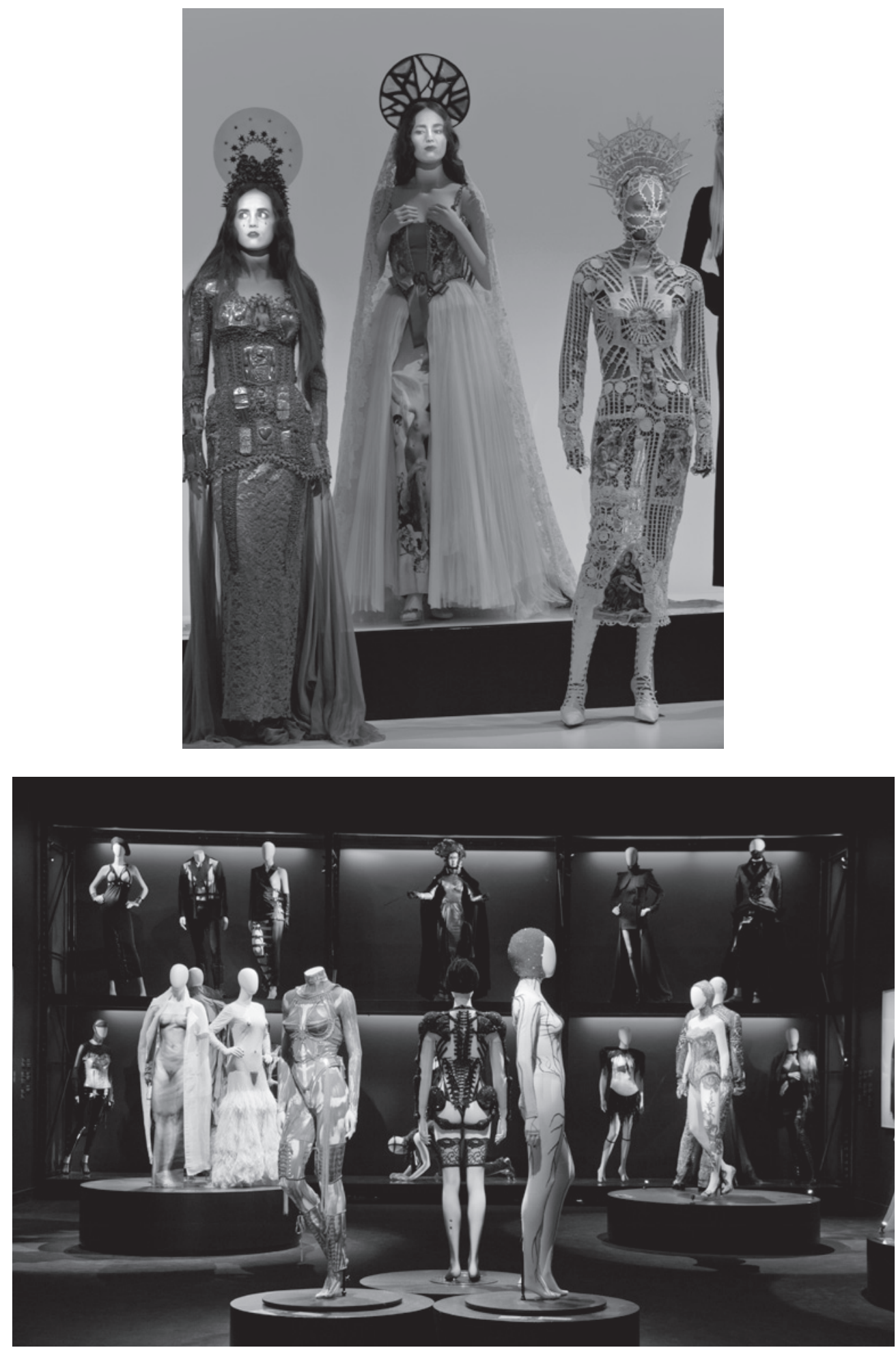

Exposition La planète mode de Jean Paul Gaultier.

De la rue aux étoiles, 2011

Scénographie : Denis Marleau (UBU) et Projectiles Photo:MBAM, Denis Farley 
en termes de droits, de technologie et d'installation: un baptême du feu. L'expérience de cette liberté iconoclaste dans la conception reste indélébile.

\section{Aujourd'hui, comment abordez-vous la conception d'une exposition?}

NB Bien entendu la rigueur et l'ambition dans la nouveauté imposent la force d'un contenu: c'est une exigence incontournable. Mais aussi la capacité à dépasser ce cadre comme la pertinence globale pour élargir les publics, et enfin la disponibilité essentielle des œuvres: ce sont trois paramètres fondamentaux. La concurrence est rude pour l'obtention des prêts, d'où l'importance d'avoir un sujet probant. Une conception bien pensée doit répondre à plusieurs interrogations préalables, car il existe une foule d'idées, mais finalement peu d'élues. Une exposition n'est pas un livre ou un inventaire plaqué sur des cimaises. Les publications scientifiques - je n'aime pas le mot "catalogue " qui réfère à des pratiques anciennes d'inventaire - obéissent à leur propre logique qui est celle de l'analyse même si, encore là, les contenus comme la mise en forme induisent une nécessaire subjectivité. Le rapprochement avec le théâtre est pour moi très signifiant par la structure, l'émotion collective partagée dans un lieu, la scénographie. Une exposition est une sélection, donc un parti pris, une architecture de l'éphémère, une première pour un corpus méconnu $(\mathrm{Cuba})$ ou une nouvelle interprétation d'un sujet (Catherine la Grande). Je m'intéresse personnellement aux expositions ouvertes, à caractère historique (notamment Cuba et Catherine la Grande), et je m'interroge sur le pouvoir des images; ce sont des problématiques très actuelles. Les exercices monographiques étant plus aisés à concevoir, je cherche à les mettre en contexte (Dix par exemple).

Cette question est aussi très personnelle. C'est toujours une histoire que je souhaite raconter, une narration dans l'espace rythmée comme une pièce de théâtre. C'est avant tout un voyage qui s'articule autour d'un désir, d'une vision intuitive. De tempérament curieux, synthétique et instinctif, j'ai le goût d'explorer des chemins de traverse inconnus: la nouveauté m'excite, c'est un fait. J'aime, imaginer des contenus, échafauder les structures, travailler les articulations, dégager les problématiques, confronter les pertinences, assembler le mot et l'image, exalter le sens et l'objet, les deux composantes qui déterminent la spécificité d'un musée. C'est un plaisir de concevoir des sujets ou des sommaires, d'éditer des livres, de penser à leur mise en page, d'imaginer des parcours, d'installer des œuvres pour que tout fasse sens et se réponde. J'espère être exigeante sur la profondeur des liens et l'audace des propositions. J'accepte facilement la critique : c'est un signe de démocratie et de pluralité. J'aime aussi m'impliquer dans le détail: scénographie et graphisme, préfaces et communiqués, textes en salles... que je rédige moi-même, ce qui étonne souvent, mais je veux garder un lien fort avec les contenus, sinon autant changer de métier! C'est d'ailleurs la raison pour laquelle je souhaitais rester conservatrice en chef. 
Quelle est votre définition en tant que conservatrice en chef d'une exposition réussie et cette définition diffère-t-elle lorsqu'on s'adresse à la directrice du musée?

NB Je dirai que la conservatrice en chef travaille sur les contenus tandis que la directrice les installe dans une vision et une dynamique institutionnelles globales.

Que pensez-vous de la "transposition thématique», de l'" apport sensoriel ", du "rapport de contraste" auxquels les œuvres sont de plus en plus assujetties dans les expositions à caractère thématique? Ces modes de médiation contribuent-ils selon vous à une interprétation, à une réception nouvelle des objets?

NB Je ne crois pas à la soi-disant neutralité de la white box: c'est une esthétique muséale formatée par le modernisme du siècle passé. Elle se prévaut d'une distanciation théorique qui aurait une valeur scientifique. Pourtant, même les sciences exactes ont du goût, comme dit Hubert Reeves. Chaque exposition, même monographique, résulte d'une sélection, donc d'une manipulation : le commissaire ou le conservateur n'est pas l'arbitre de son jeu, il en est le principal joueur. La comparaison avec un chef d'orchestre, qui interprète toujours une partition selon sa sensibilité, est en ce sens éclairante. L'histoire de l'art a parfois le complexe de ne pas s'afficher comme une science et certains spécialistes font appel aux œuvres comme à des illustrations de leurs commentaires: je suis une "conservatrice» de musée (un nom de métier très réducteur, mais soit...), donc la présence concrète des œuvres m'importe et me parle; elle ne peut être réduite à la platitude numérique de figures de textes. Il me semble que la connaissance sensible portée par l'expérience physique est un terrain à explorer car les œuvres sont créées pour parler aux sens : il y a un transport d'émotion qui génère de la réflexion. C'est pourquoi le décloisonnement des disciplines me semble fertile en dialogues.

Prenons garde à ne pas amputer les œuvres qui parlent un langage bien plus complexe que le nôtre. Il s'agit néanmoins d'une liberté sous conditions qui doit répondre à une articulation intellectuelle sans gratuité. Forcément, une exposition temporaire autorise plus de liberté qu'une exposition permanente faite, comme son nom l'indique, pour durer. L'instrumentalisation est donc inévitable, mais quel que soit le filtre choisi, il doit être expliqué : cette approche est plus créative, stimulante, les contrastes s'avérant complémentaires. Comparer une même exposition d'un musée à l'autre révèle en fait des différences conceptuelles avec des écarts étonnants : c'est un sujet qui me passionne, car il montre justement la pluralité de ces approches. Une partie de mon travail consiste d'ailleurs à penser ou à repenser les projets en fonction de "notre histoire».

Vous avez présenté le travail de créateurs de mode: Yves Saint Laurent et Jean Paul Gaultier. Ces expositions, qui ont accaparé autant les médias que le public, ont eu une fortune critique plus que positive. Que représente donc ce type de production pour vous? 
NB Si les expositions consacrées à la mode sont actuellement en vogue, McQueen à New York ayant cristallisé un phénomène parfois opportuniste, je considère que l'ouverture du MBAM à la couture se justifie par le renforcement du pôle design, avec notre nouveau pavillon d'arts décoratifs, comme par l'importance incontestable de nos collections à l'échelle continentale. Même si je n'envisage pas de former une collection de mode au musée - à moins d'une donation exceptionnelle !-, j'ai voulu engager un spécialiste, qui s'intéresserait aussi à la photographie de mode plus pertinente pour notre fonds.

Par ailleurs, je crois que les expositions doivent montrer ce qui n'est pas accessible au public: or il est plus facile de voir un tableau de Van Gogh qu'une robe de haute couture! Après avoir défilé quelques minutes sur un podium devant un rare public, elle disparaît. Il y a confusion entre médiatisation et exposition : la surabondance d'images rend l'univers de la couture faussement familier, pourtant seule l'expérience live permet de saisir la virtuosité de cette confection de l'excellence. Je milite pour la reconnaissance du design à côté des beaux-arts, le mercantilisme qui leur est reproché étant, à mon avis, hors de propos, surtout quand les stratégies du marché de l'art contemporain n'y échappent pas non plus. Certains sont des artisans de l'extrême, des funambules qui portent leur vie sur un fil, soumettant leur créativité à une concurrence impitoyable suivant la cadence implacable des saisons. Il y a peu d'artistes qui vivent une telle pression. L'univers de paillettes des grandes maisons de luxe nous aveugle, mais pour les couturiers indépendants, je pense à Denis Gagnon qui a été présenté au musée, et même à Jean Paul Gaultier j'en étais étonnée -, le succès ou non de chaque saison rappelle la vulnérabilité de leur situation économique.

Enfin, et surtout, ce qui m'attire dans mes choix est la force d'un imaginaire, qui saura transcender le champ disciplinaire pour porter son art au-delà, peu m'importe qu'il soit cinéaste, couturier ou peintre: le médium n'est pas le message.

Les musées d'art ont toujours eu une connotation élitiste, et ce type d'exposition appelait de toute évidence un élargissement du public. De quels autres moyens un musée comme le vôtre dispose-t-il pour rejoindre une clientèle qui n'est pas nécessairement fidélisée?

NB Même si l'accessibilité reste un enjeu primordial, les musées sont de moins en moins élitistes: la popularité des manifestations culturelles le démontre dans nos sociétés davantage scolarisées et informées. À la différence des grands musées de Paris ou de New York, visités aux trois quarts par des touristes, le MBAM reçoit la même proportion de visiteurs... mais de sa région métropolitaine! Le défi est donc de les faire revenir plusieurs fois dans l'année. Comme l'offre culturelle montréalaise est surabondante, il est impératif de diversifier mes stratégies: saisons gratuites autour de grandes expositions fédératrices (Pour l'art!, Imagine, Big Bang...), renforcement de la multidisciplinarité, notamment 
avec la musique et la mode, travail sur les contenus en profondeur pour exalter leur richesse en ouvrant vers le champ «civilisationnel», enfin amener les sujets vers des problématiques ou des référents actuels.

C’est pourquoi j'ai toujours souhaité renforcer le rôle "politique " de l'institution dans la cité, au premier sens du terme, pour que nos projets portent des valeurs au-delà du champ plus sectaire de l'histoire de l'art. Même si la pertinence intellectuelle, la prise de risque calculé et la nouveauté des contenus (sujet, publication, scénographie) demeurent des fondamentaux dans les choix de programmation, cela ne suffit pas. Il faut tenter de réussir ce grand écart entre le niveau "macro", qui établit à une échelle internationale la notoriété des expositions, souvent des productions maison que nous exportons à l'étranger, et le niveau «micro ", c'est-à-dire notre public local qui doit pouvoir associer la pertinence des contenus à sa vie, à ses préoccupations, à une actualité commune. Je me méfie des commissaires qui, au mépris des publics, ne parlent qu'à leur «club». C'est une erreur de jugement, car le public, même non spécialiste, est souvent instruit.

Pour les expositions Cuba, Imagine, Otto Dix, ma volonté politique était évidente. Tiffany permettait de raccrocher la nouveauté d'une recherche scientifique à la découverte d'un patrimoine commun, les vitraux de l'église Erskine \& American étudiés, restaurés et révélés pour l'occasion. Parfois, les liens sont davantage construits : c'était le cas de Grandeur Nature, qui mettait en exergue un message écologique, ou de Van Dongen, qui se raccrochait à l'héritage de Max Stern, ou encore de Waterhouse, qui s'appuyait sur un double héritage esthétique français et anglais commun proche des racines montréalaises : je suis extrêmement sensible à conceptualiser ces liens le plus possible.

Dans le même esprit, je défends les projets fédérateurs qui transforment le musée en une plateforme de rencontres: Imagine et Big Bang ont fonctionné comme des open works. Ces expériences risquées ont été fructueuses, passionnantes, beaucoup plus profondes et complexes qu'elles ne le laissent penser. La gratuité de ces grandes manifestations est une valeur efficace pour porter un message le plus largement possible: rapprocher les Montréalaises et les Montréalais de leur histoire dans Les Sulpiciens, les collectionneurs et les visiteurs dans Pour l'art!, les œuvres de notre collection et les artistes de toutes disciplines dans Big Bang, l'art le plus avant-gardiste et le public avec E-Art... Depuis 1995, le musée est généreux en donnant l'accès gratuit à sa collection permanente - comme le MNBAQ plus récemment- une rareté pour les grands musées pancanadiens! J'ai élargi cette politique aux expositions d'art contemporain. Mais attention, la gratuité a un prix que nous assumons : c'est une forte conviction personnelle. 


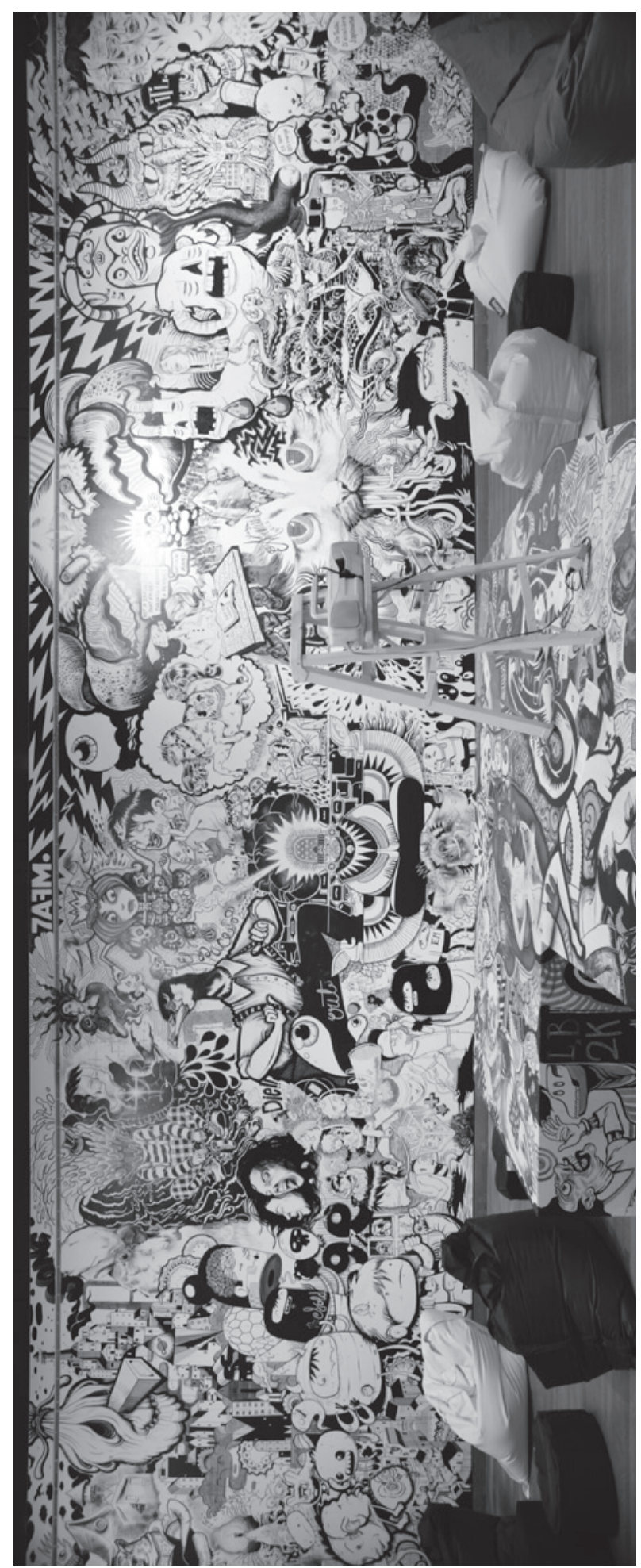

Exposition Big Bang.

Carte blanche à la créativité, 2011

Installation Collectif En Masse

Photo: Marc Cramer 


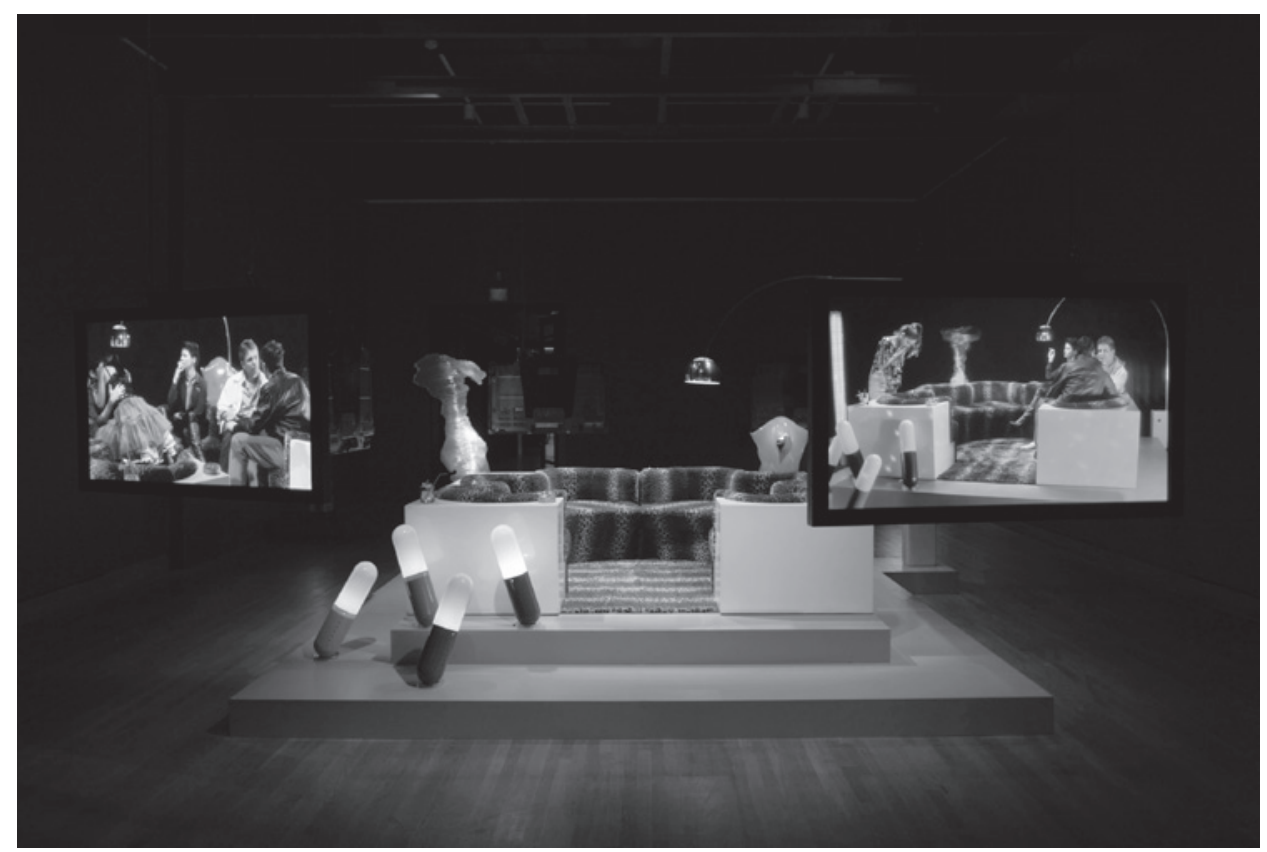

Exposition Big Bang.

Carte blanche à la créativité, 2011

Installation Adad Hanah et Denys Arcand

Photo: Marc Cramer

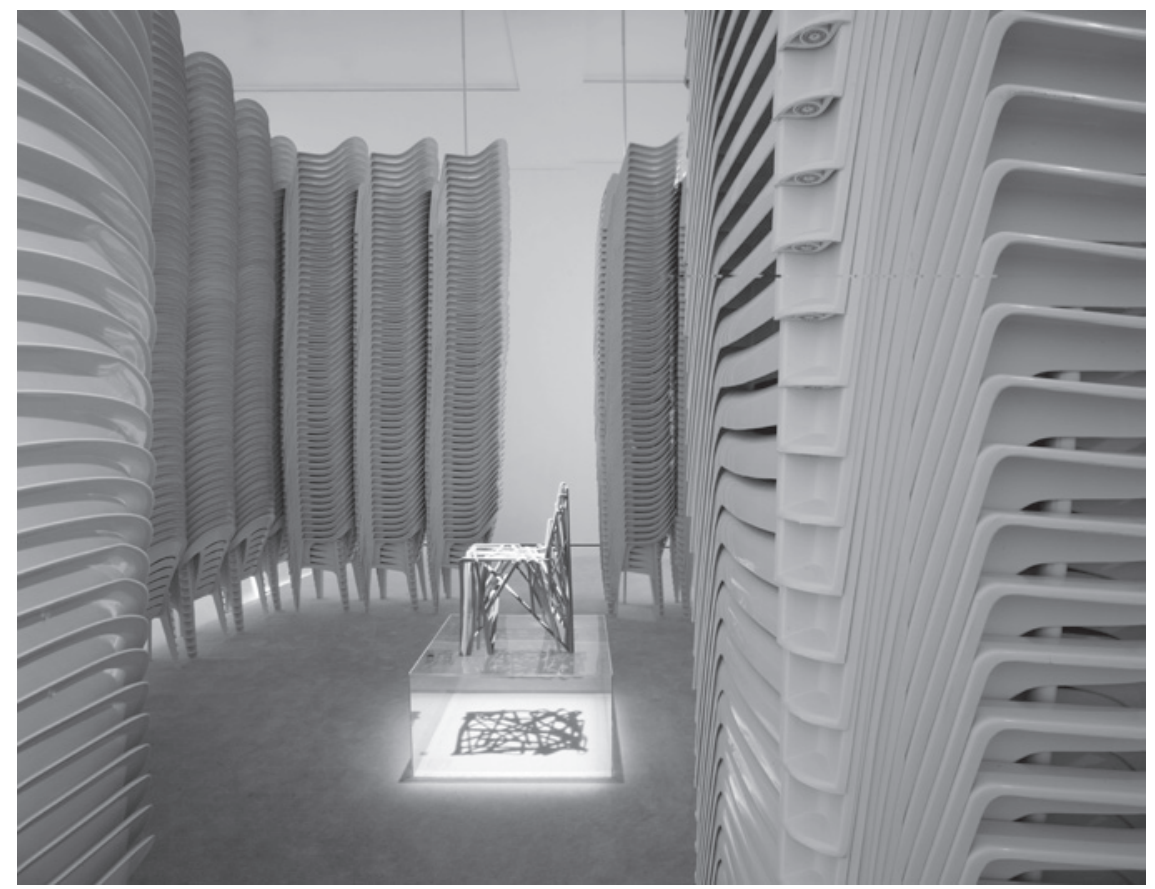

Exposition Big Bang.

Carte blanche à la créativité, 2011

Installation Patrick Jouin / Pierre Lapointe

et Jean Verville

Photo: Marc Cramer 


\section{Depuis votre entrée en fonction le musée semble vouloir définitivement marquer de son empreinte le tissu culturel; selon vous, comment se positionne-t-il face à ses homonymes québécois, canadiens et internationaux?}

NB Plus qu'un compétiteur, je souhaite que le musée soit considéré comme un partenaire, la collaboration étant l'avenir de nos sociétés, une solution économique et fédératrice à tous les niveaux d'échange. Contre un certain esprit de forteresse, nous pouvons travailler ensemble au niveau municipal. L'avenir est aux grandes régions urbaines dont la concurrence se renforce au moment où le pouvoir et l'aide financière des États fatalement diminuent. Si Montréal n'est pas une capitale gouvernementale, elle est la métropole culturelle qui a tout avantage à faire travailler en synergie ses institutions : je crois beaucoup au rayonnement concentrique.

Par ailleurs, le MBAM est le premier musée d'art au Québec et l'un des plus importants au Canada. Cette constatation - dont je ne tire aucune fierté - s'explique simplement par l'ampleur et la richesse de ses collections. C'est aussi une institution reconnue pour la créativité et le dynamisme de sa programmation, souvent exportée à l'international. Cet héritage précieux pour l'ensemble du pays impose néanmoins la vigilance, non pas à cause d'une compétition stérile entre les musées, car je crois, au contraire, cette stimulation bénéfique pour la diffusion d'une cause commune, mais plutôt en raison d'une profonde métamorphose des référents culturels. C'est pourquoi nous ouvrons un grand chantier pour l'éducation qui prolongera notre action vers la relève : à quoi bon de belles galeries si elles restent vides demain?

Que pouvez-vous nous dire à propos des futurs projets du musée? NB L'éducation est une de mes grandes priorités. La réinvention du MBAM nous amène au milieu du pont; bien du chemin reste à parcourir pour rejoindre davantage de clientèles scolaires et universitaires, familiales et communautaires. Les objectifs sont ambitieux pour le musée; j'espère qu'ils le seront tout autant pour le plan d'action des ministères de la Culture et de l'Éducation, afin de mieux promouvoir les arts, mais surtout l'histoire, auprès des écoliers. J'appelle de tous mes vœux cet enseignement sur les bancs d'école. Ce n'est pas une volonté portée par un sentiment protectionniste, mais bien la nécessité de fournir des référents culturels et des outils d'objectivation contre l'aliénation médiatique qui nous submerge. Après tout, le rôle d'un musée est avant tout de conserver pour transmettre: nos civilisations sont mortelles, déclarait Paul Valéry en 1919.

Nous disposons dorénavant davantage d'outils efficaces pour promouvoir, au-delà de la collection, plus que l'histoire de l'art : une histoire essentielle et fondatrice. Mais quid de belles galeries ou de livres scientifiques... sans visiteur ni lecteur? C'est pour cette raison que notre prochain grand chantier est l'éducation à tous les niveaux: scolaire, universitaire, adultes, avec des objectifs ambitieux; nous souhaitons accroître les visites scolaires des 40000 écoliers par an à 100000 , travailler en amont avec les commissions scolaires et les universités 
pour offrir des contenus plus adaptés, ainsi que des prix et des bourses. Cet enjeu est essentiel, notamment pour perpétuer une histoire patrimoniale du Québec, de plus en plus délaissée au profit des arts actuels: c'est d'ailleurs un problème très sérieux que j'aborde dans l'introduction de notre publication sur l'art québécois et canadien.

Le tourisme est aussi, bien entendu, un objectif à développer, mais n'oublions pas que les trois quarts de notre clientèle viennent de la région métropolitaine. Notre membership a récemment augmenté de manière exponentielle avec, à ce jour, plus de 61000 amis, mais qu'en sera-til après-demain? Dans la déferlante médiatique qui nous inonde, les musées doivent conserver une position critique comme outil d'objectivation essentiel à notre formation: la tyrannie des images nous domine plus que jamais; les impératifs esthétiques, par exemple, nous en donne des preuves affligeantes chaque jour. C'est plus grave quand la propagande idéologique, au Canada même, recourt aux images dans une indifférence inquiétante. Cela est très vrai pour une histoire identitaire - je n'ai pas peur du mot d'autant plus que je suis une immigrée -, mais c'est aussi vrai pour une histoire de l'art en déficit d'apprentissage scolaire dans une majorité de pays : cette problématique rend le Québec doublement vulnérable.

\section{Pour conclure, que souhaitez-vous à court et à long terme pour} le Musée des beaux-arts de Montréal?

NB Comme la musique, les œuvres doivent être vues pour pleinement exister. Aucun enregistrement ne remplacera l'expérience de l'écoute en concert; aucune reproduction ne se substituera à la rencontre physique avec les œuvres. Dans un univers où la banalisation de l'image élargit mais appauvrit notre rapport à l'art, je souhaite promouvoir une culture live, cette émotion incompressible à la reproduction où le musée agit comme véhicule d'une indispensable connaissance sensible sur le monde, avec des collections vivantes, animées par la pluralité des flux de la vie. 